\title{
An investigation of the sensitivity of the clear-sky outgoing longwave radiation to atmospheric temperature and water vapor
}

\author{
Yi Huang, ${ }^{1}$ V. Ramaswamy, ${ }^{2}$ and Brian Soden ${ }^{3}$ \\ Received 21 November 2005; revised 19 September 2006; accepted 30 October 2006; published 3 March 2007.
}

[1] The rate at which the outgoing longwave radiation (OLR) responds to perturbations in temperature and moisture plays a fundamental role in determining climate sensitivity. This study examines the clear-sky OLR sensitivities to temperature and water vapor, as quantified by its partial derivatives (radiative Jacobians). The Jacobians, as computed by the Geophysical Fluid Dynamics Laboratory (GFDL)'s line-by-line (LBL) radiative transfer model are used to verify the results from the parameterized GFDL GCM (general circulation model) radiation code. The results show that the (1) Jacobians of OLR due to incremental changes in temperature and water vapor are insensitive to different formulations of water vapor continuum absorption and (2) Jacobians of OLR are properly captured by the GCM longwave band approximation. Simulations with the GCM demonstrate that uncertainties in the formulation of continuum absorption have little impact on the climate model simulation of clear-sky OLR changes in response to prescribed sea surface temperature (SST) perturbation. The numerically computed Jacobians of OLR are used to reconstruct the tropical annual mean OLR from the variations of temperature and water vapor over the period 1980-1999. The reconstructed OLR anomaly time series agrees well with that computed explicitly by the GCM. On the basis of this result, it becomes possible to separate out the temperature and water vapor contributions to the OLR variation. The results show that the temperature contribution dominates the water vapor contribution in the lower and middle troposphere, while in the upper troposphere the two contributions largely offset each other.

Citation: Huang, Y., V. Ramaswamy, and B. Soden (2007), An investigation of the sensitivity of the clear-sky outgoing longwave radiation to atmospheric temperature and water vapor, J. Geophys. Res., 112, D05104, doi:10.1029/2005JD006906.

\section{Introduction}

[2] Many previous studies have highlighted the role of water vapor in regulating the radiative damping of climate perturbations (see Held and Soden [2000] for a review). It has also been recognized that water vapor's effect on the outgoing longwave radiation (OLR) is spectrally and height-dependent [Shine and Sinha, 1991; Clough et al., 1992; Slingo and Webb, 1997] and varies geographically [Raval et al., 1994; Bony and Duvel, 1994; Allan et al., 1999]. Modeling studies, e.g., Zhang et al. [1994], note that water vapor's effect on OLR in response to a climate change is highly coupled to temperature, with these two opposing feedbacks largely offsetting each other. Although it is believed that the uncertainty in the model simulated climate sensitivity is mostly due to clouds [Cess et al., 1990, 1996], the apparent agreement in clear-sky sensitivities might

\footnotetext{
${ }^{1}$ Program in Atmospheric and Oceanic Sciences, Princeton University, Princeton, New Jersey, USA.

${ }^{2}$ NOAA Geophysical Fluid Dynamics Laboratory, Princeton, New Jersey, USA.

${ }^{3}$ Rosenstiel School for Marine and Atmospheric Science, University of Miami, Miami, Florida, USA.

Copyright 2007 by the American Geophysical Union. 0148-0227/07/2005JD006906
}

comprise a subtle cancellation due to different water vapor and temperature feedbacks [Allan et al., 2002].

[3] To a first order approximation, the climatic feedback effect of a particular variable $(X)$, measured by the TOA (Top of the Atmosphere) radiative flux variation $(\Delta R)$, is the product of its climatic response $(\Delta X)$ and the TOA radiative sensitivity to this variable $\left(\frac{\partial R}{\partial X}\right)$. Although both climatic response and radiative sensitivity are critical in determining the climate change, we are especially concerned with the latter factor, $\frac{\partial R}{\partial X}$, in this paper. Radiative transfer codes were examined in the ICRCCM project [Ellingson and Fouquart, 1991] with regards to the simulation of radiation fluxes and radiative cooling/heating rates under various atmospheric conditions. In this study, we extend this comparison by considering the simulated TOA radiative sensitivity, which gives a more stringent criterion for the evaluation of the radiation code for computing climate feedbacks. Specifically, we focus on the clear-sky OLR, particularly its sensitivity to atmospheric water vapor and temperature.

[4] As a basis for the following analysis, we first present a LBL-based investigation concerning the spectral dependence of clear-sky OLR on the vertical distributions of 
water vapor and temperature, emphasizing their relative roles. As an example, a practically relevant case postulating constant relative humidity $(\mathrm{RH})$ to link water vapor and temperature changes is studied here. Then, the Jacobians obtained from the GCM radiation code are compared to those obtained from LBL calculations. Uncertainties due to water vapor continuum absorption formulation and longwave band approximation are carefully examined, including a series of GCM experiments to directly quantify its impact on climate sensitivity. Lastly, as an application, it is shown that the Jacobians can be used to decompose interannual anomalies in OLR into the individual contributions arising from variations in water vapor and temperature.

\section{Quantification of Clear-Sky OLR Sensitivity}

\subsection{Method}

[5] For a plane-parallel atmosphere, the clear-sky OLR is composed of both emission from the surface and the contributions from consecutive atmospheric layers, and thus is a function of many variables such as surface temperature, atmospheric temperatures and absorption gases' concentration and distribution [Liou, 1980]. Mathematically this can be written as an implicit function of these variables,

$$
O L R=f\left(T_{s}, T_{a}, q, \ldots\right),
$$

where $T_{s}$ is surface temperature; represents the atmospheric temperature profile; $T_{a}$ and $q$ represents the water vapor mixing ratio profile.

[6] With a Taylor expansion, the change of OLR can be expressed as (here we focus on the variation of temperature and water vapor profiles while fixing all the other variables),

$$
\begin{aligned}
\Delta O L R= & \frac{\partial f}{\partial T_{s}} \Delta T_{s}+\sum_{i} \frac{\partial f}{\partial T_{a}^{i}} \Delta T_{a}^{i}+\sum_{i} \frac{\partial f}{\partial q^{i}} \Delta q^{i} \\
& + \text { Residual } \ldots,
\end{aligned}
$$

where $\mathrm{i}$ is an index of vertical levels.

[7] If the change in these variables is small enough, the residual term can be neglected. The partial derivatives of OLR with respect to the variables, for example, $\frac{\partial f}{\partial T_{a}^{i}}\left(\mathrm{~W} \mathrm{~m}^{-2} /\right.$ unit change of temperature $)$ and $\frac{\partial f}{\partial q^{i}}\left(\mathrm{~W} \mathrm{~m}^{-2} / \mathrm{unit}^{i}\right.$ change of water vapor), are used to quantify the OLR sensitivity in this paper. These derivatives are hereinafter referred to as the Jacobians.

[8] In order to calculate these partial derivative terms, we perturb the standard profiles, one layer at a time, calculate the OLR with the column radiative transfer models, and obtain the Jacobians by differencing. The radiative transfer models used here are GFDL's LBL model [Fels and Schwarzkopf, 1981; Schwarzkopf and Fels, 1985; Schwarzkopf and Ramaswamy, 1999], and a band model based on the Simplified Exchange Approximation (SEA) [Fels and Schwarzkopf, 1975; Schwarzkopf and Fels, 1991; Schwarzkopf and Ramaswamy, 1999]. The latter is also the longwave radiation code used in the GFDL GCMs [Global Atmospheric Model Development Team (GAMDT), 2004]. Both models take into account the longwave absorbing species, including $\mathrm{H}_{2} \mathrm{O}, \mathrm{CO}_{2}, \mathrm{O}_{3}, \mathrm{CH}_{4}$, $\mathrm{N}_{2} \mathrm{O}$, CFC-11, CFC-12, CFC-113 and HCFC-22. In this study, both models have 25 vertical levels, i.e., the same resolution as the GFDL GCM. (A comparison with computations using a finer 41-level resolution confirms the essential results found here.) For temperature profiles, the perturbation increment is $1 \mathrm{~K}$ at each model layer. For water vapor profiles, the perturbation increment is $10 \%$ increase of mixing ratio at each layer. We have examined the validity of the Taylor expansion used to define the sensitivity. For a few degrees' change of temperature and up to $20 \%$ change of water vapor mixing ratio at each model layer, the sum of the differential terms on the right hand side of equation (1), reproduces the change of OLR as directly simulated from the radiative transfer models. Other studies, such as Shine and Sinha [1991] and Fasullo and Sun [2000], have also showed that the OLR change can be obtained by linearly summing the differentials.

\subsection{Numerical Results}

[9] Figure 1 shows the LBL-calculated Jacobians for three standard atmospheres, tropics (TRP), midlatitude summer (MLS) and midlatitude winter (MLW) [McClatchey et al., 1972]. The absolute values of OLR change have been scaled to correspond to perturbations at each 50 mbar thick layer. The frequency-integrated Jacobian curves of water vapor agree with Shine and Sinha's [1991] results, even though a spectrally finer LBL model with a newer version of Clough's water vapor continuum absorption scheme [Tobin et al., 1999] is used here. These curves for different atmospheres differ substantially in terms of their shapes and sensitivity values. The TRP, MLS and MLW curves show decreasing sensitivity values in order. The TRP curves have a prominent peak in the lower troposphere; the MLS curves weight the lower and upper parts of the troposphere nearly equally; the MLW curves maximize in the upper troposphere. Comparison between the temperature and water vapor Jacobians shows that the OLR change caused by $10 \%$ perturbation in water vapor mixing ratio is roughly the same magnitude as that caused by $1 \mathrm{~K}$ perturbation in temperature. However, exceptions exist in the layers close to the surface and in the stratosphere, where the Jacobians of water vapor approach zero while the Jacobians of temperature are large.

[10] We further decompose the total OLR flux change into contributions from different spectral regions, which provides insights into the above features. Taking the tropical case as an example, Figures $2 \mathrm{a}$ and $2 \mathrm{~b}$ illustrate the change of outgoing flux in each $1 \mathrm{~cm}^{-1}$ spectral range in response to height-dependent water vapor and temperature variations. These spectral Jacobians (distinguished from the frequencyintegrated Jacobians in Figure 1) also agree with Shine and Sinha's result.

[11] These two spectral figures illustrate how different absorption bands contribute to the OLR sensitivity and account for the features shown in Figure 1. Because the absorption in rotation band is saturated in terms of the radiation from the surface and lower troposphere, the outgoing radiation flux below $650 \mathrm{~cm}^{-1}$ shows no sensitivity to the surface and lower troposphere. The strong absorption in the $\mathrm{CO}_{2} \nu 2$ band (around $670 \mathrm{~cm}^{-1}$ ) makes the outgoing radiation between 620 and $720 \mathrm{~cm}^{-1}$ completely insensitive to the water vapor variation, but very sensitive to the temperature in the lower stratosphere. Water 
(a) Irradiance Jacobians of $\mathrm{H} 2 \mathrm{O}$

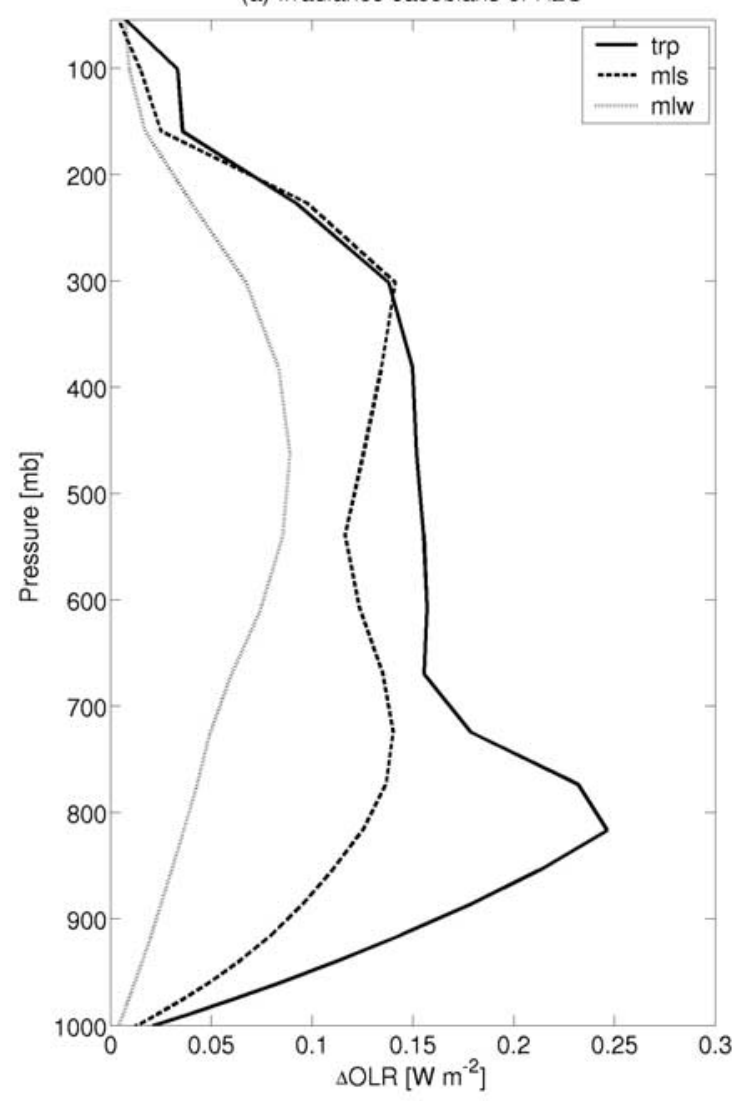

(b) Irradiance Jacobians of Temperature

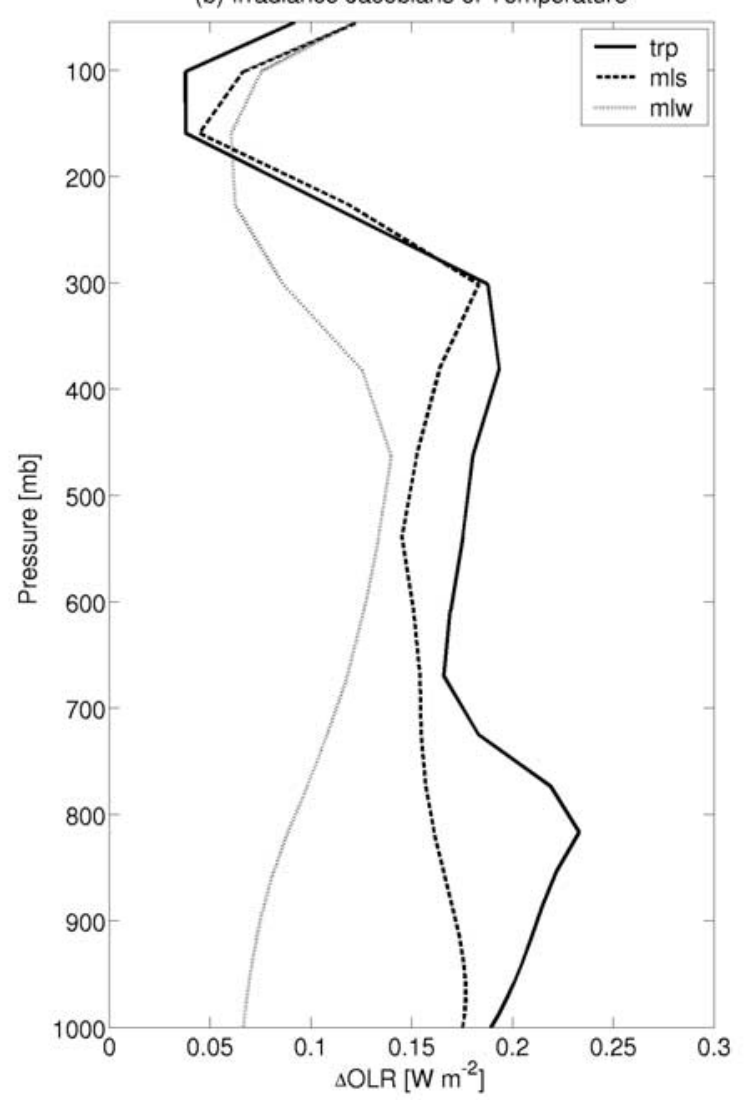

Figure 1. Frequency-integrated Jacobians of (a) water vapor and (b) temperature. The Jacobians are calculated using the GFDL LBL model for three standard atmospheres, tropics (TRP), midlatitude summer (MLS) and midlatitude winter (MLW). The absolute value of the Jacobians is plotted.

vapor continuum absorption in the window region (800 to $1200 \mathrm{~cm}^{-1}$ ) accounts for the sensitivity to the lower troposphere. When water vapor is abundant, as in the tropics, the continuum absorption becomes important enough to cause a large peak in the lower troposphere. Note in the window region, for the layers close to the surface, the sensitivity to temperature is much larger than that to water vapor. There is a noticeable damping of the sensitivity to lower troposphere at around $1050 \mathrm{~cm}^{-1}$ in both figures; this is due to the strong absorption by $\mathrm{O}_{3}$ in the stratosphere. The water vapor $\nu 2$ band (vibration-rotational band), located around $1600 \mathrm{~cm}^{-1}$, is also strong enough to make the outgoing radiation in this region insensitive to the surface and the lower atmosphere but very sensitive to the upper troposphere. Because the Planck function at terrestrial temperatures has relatively small values in the water vapor $\nu 2$ band, the spectral Jacobians are of rather small values in this region. However, in terms of the relative change (normalized by the actual outgoing radiation), the Jacobians values would in fact be very large [Slingo and Webb, 1997].

[12] The spectral Jacobian plots are similar to the spectrally decomposed atmospheric contribution to OLR, illustrated by Clough et al. [1992], except in the $\mathrm{CO}_{2}$ and $\mathrm{O}_{3}$ bands which were not included in their calculation. This reflects the fact that the outgoing spectral radiance is most sensitive to the atmospheric layer where most of the radiant energy originates.

\subsection{Constant RH Case}

[13] The manner in which the moisture profile is perturbed may result in different shapes of the frequencyintegrated Jacobian curves. In Figure 1, in which a vertically uniform fractional perturbation of mixing ratio was imposed, the tropical curve yields a much larger sensitivity in the lower troposphere than in the upper troposphere. In contrast, Spencer and Braswell [1997] perturbed the water vapor profile by an equal increment of relative humidity. Because the relative humidity tends to decrease with height, this produces much larger fractional changes in mixing ratio in the upper troposphere than in the lower troposphere. As most models tend to conserve relative humidity as the climate warms [Held and Soden, 2000], we also consider this case here.

[14] Perturbing the temperature profile by $1 \mathrm{~K}$ while simultaneously keeping the RH constant will yield a change in OLR that can be attributed to contributions from both temperature and water vapor. Figure 3 illustrates the results for the tropical atmosphere. The OLR at TOA is most sensitive to the temperature change in the bottom part $(\mathrm{P}>$ 800 mbar) of the atmosphere; above this altitude $(\mathrm{P}<$ 

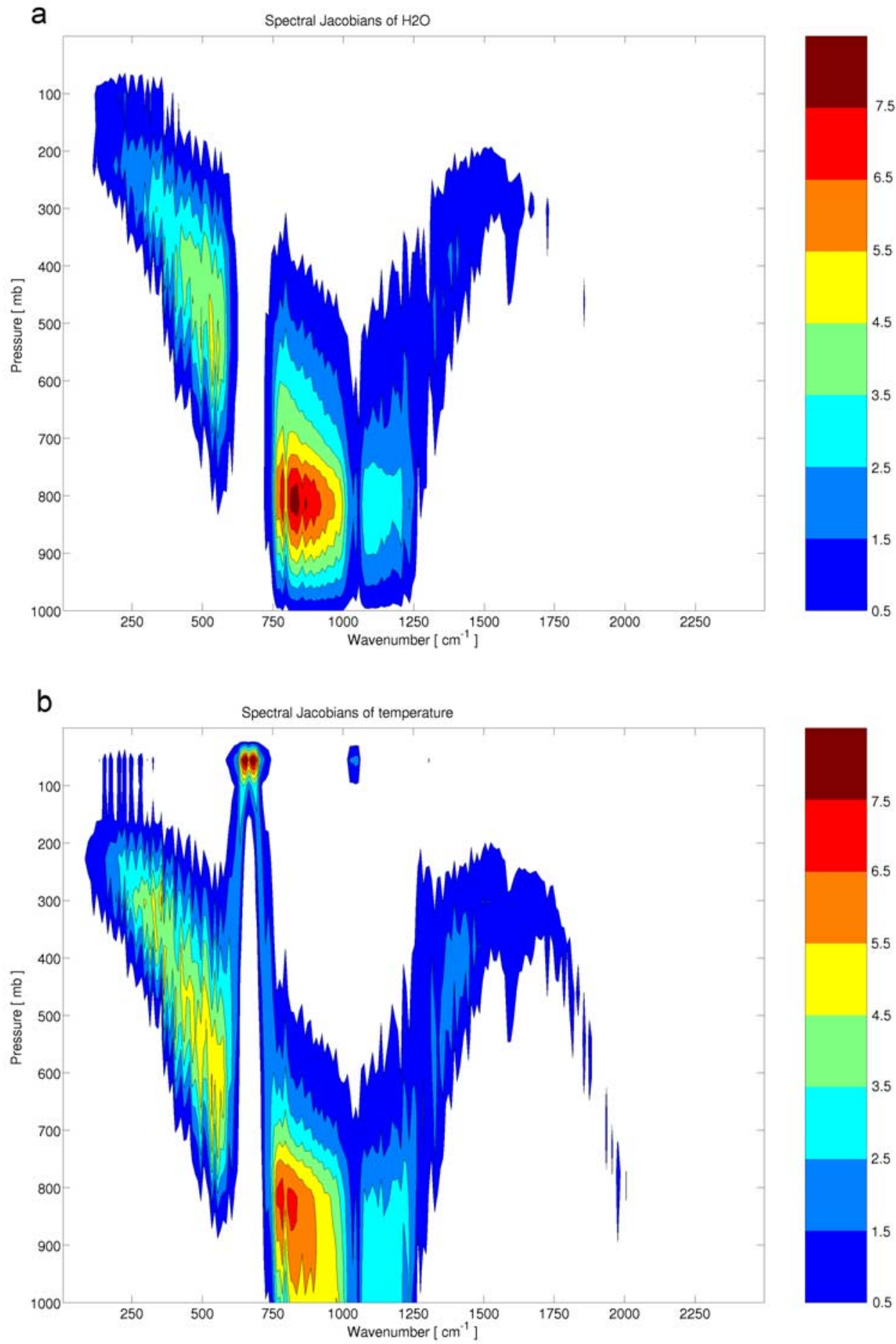

Figure 2. Spectral Jacobians for tropical atmosphere. Absolute values of OLR change due to (a) $10 \%$ water vapor mixing ratio perturbation, where contour interval is $1 \times 10^{-4} \mathrm{~W} \mathrm{~m}^{-2} \mathrm{per} \mathrm{cm}^{-1}$, and (b) OLR change due to $1 \mathrm{~K}$ temperature perturbation.

800 mbar), the opposing contributions by water vapor and temperature largely cancel each other. The OLR change is positive when temperature and water vapor both increase under the constant RH assumption, though an exception occurs near the tropopause (between 100 and 200 mbar). For the midlatitude summer and midlatitude winter atmos- pheres, we obtain similar results, with the temperature contribution prevailing against the water vapor contribution. This indicates that, under assumption of constant $\mathrm{RH}$ response, only in the upper most portion of the troposphere can the increased opacity from the moistening exceed the damping effect due to temperature increase. 


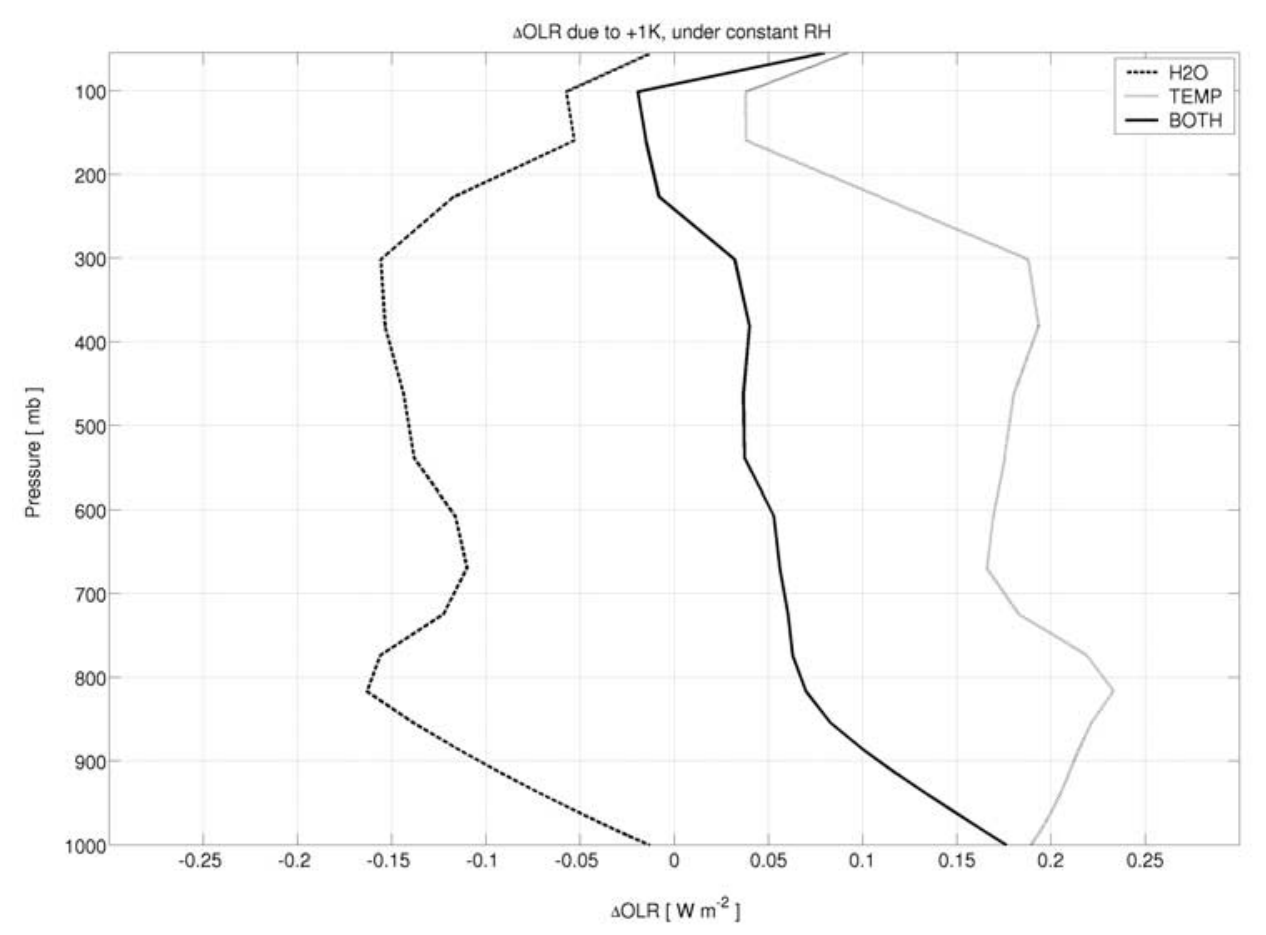

Figure 3. OLR change due to temperature (TEMP), water vapor (H2O), and simultaneous changes in both factors (BOTH), as calculated for the tropical atmosphere. "TEMP" represents the change of OLR due to $1 \mathrm{~K}$ temperature perturbation in each 50 mbar thick layer, " $\mathrm{H} 2 \mathrm{O}$ " is the OLR change due to the resultant water vapor change in each layer while conserving the relative humidity, and "BOTH" is the overall change due to both water vapor and temperature. All three curves are derived from the LBL calculation.

[15] In this tropical case (Figure 3), it is also noted that, in contrast to a single peak in either lower [see Shine and Sinha, 1991] or upper troposphere [see Spencer and Braswell, 1997], the water vapor curve weights the lower and upper parts of troposphere almost equally. This implies that the two parts have comparable importance in terms of the water vapor feedback. Note this result is obtained when considering a vertically uniform temperature perturbation and assuming that $\mathrm{RH}$ is conserved. The tendency of the climate system to conserve $\mathrm{RH}$ is manifest in several modeling studies [Held and Soden, 2000]. This assumption is used here simply to provide a reasonably realistic constraint on water vapor change associated with temperature change. As for temperature perturbation, both observational and modeling studies [e.g., Gillett et al., 2000] suggest that the magnitude of temperature change is larger in the upper troposphere. This factor would make the upper troposphere contribute more to the OLR change than the lower portions of the troposphere, as to be illustrated in section 4. The masking effects of clouds on the OLR also make the upper tropospheric contribution more significant in the case of an "all-sky" atmosphere.

\section{Computational Uncertainties}

[16] In order to address the climate change problem, climate models must accurately simulate the climate sensitivity at TOA. With the above developed method of radiative Jacobians, we examine two prominent issues that might cause uncertainties in numerical quantification of the clearsky OLR sensitivity: (1) empirical formulation of water vapor continuum absorption and (2) the longwave band approximation adopted in climate models. Then in a series of GCM simulations their combined effect on the model simulated climate sensitivity is examined.

\subsection{Water Vapor Continuum Absorption}

[17] As noted by Clough et al. [1992] and Held and Soden [2000], water vapor continuum absorption is one of the major sources of uncertainty in radiative transfer calculations. Because of the lack of a complete theoretical explanation, this part of absorption is represented by semiempirical formulae constrained by laboratory and atmospheric measurements. The formulae usually account for one or both of the two possible causes of the continuum absorption, i.e., self-broadening (collision between water vapor molecules) and foreign-broadening (collision between water vapor molecule and other gas molecules). We investigate the effects of three different continuum formulations that have been used in GFDL as well as other climate models. These formulations are the Roberts scheme [Roberts et al., 1976] (hereinafter RSB) and two versions of the Clough scheme [Clough et al., 1989; Tobin et al., 1999] (hereinafter CKD2.1 and CKD2.4). In RSB, only self-broadening is considered, while the Clough scheme includes both self- and foreign- broadenings. CKD2.1 and CKD2.4 differ only with regard to the parameterization in the rotation band. For the sake of comparison, we add a 
(a) TRP

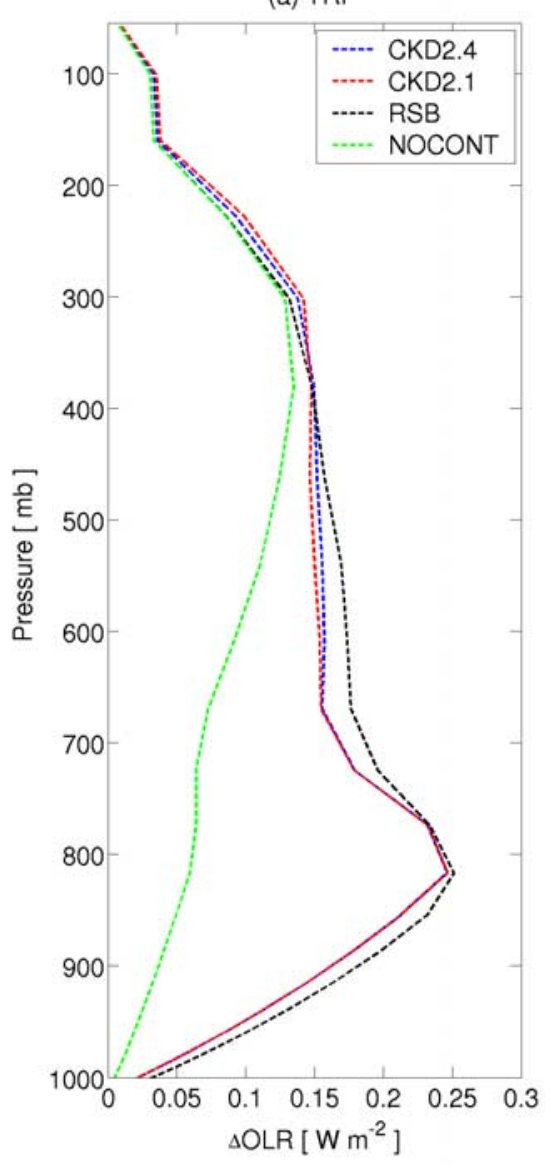

(b) MLS

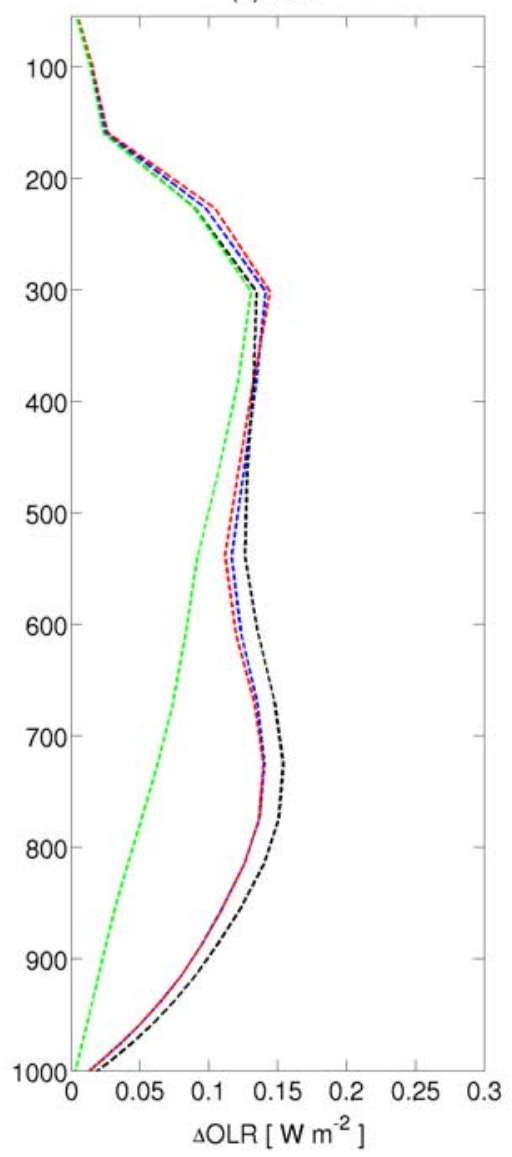

(c) MLW

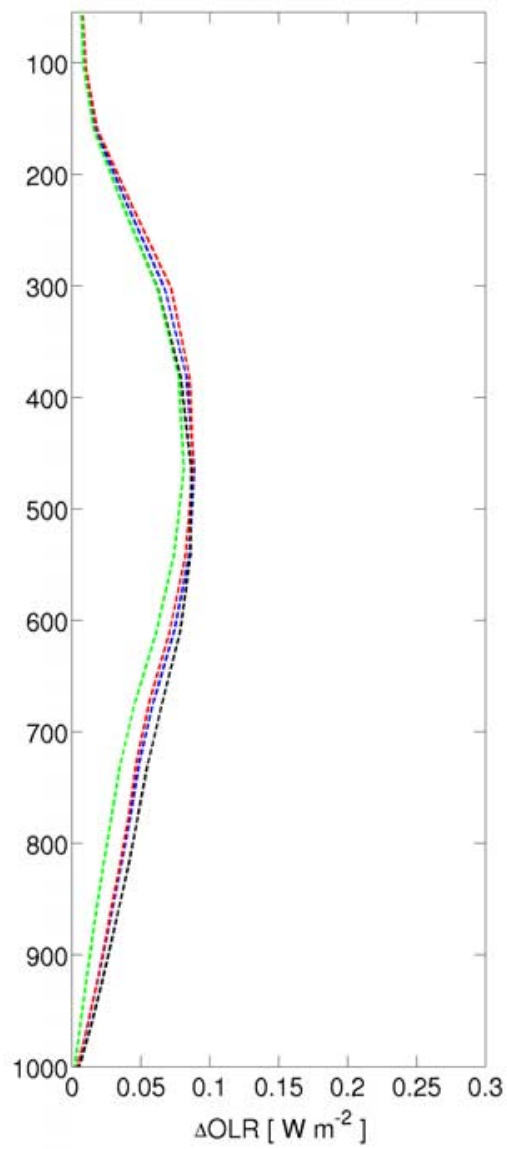

Figure 4. Jacobians of water vapor calculated with the GFDL LBL model for different formulations of water vapor continuum absorption, namely, CKD2.4, CKD2.1, RSB, and NOCONT (see text). (a) TRP, (b) MLS, and (c) MLW atmospheric profiles are considered.

fourth case, in which no continuum absorption is included (hereinafter NOCONT). This is a hypothetical case that enables the fundamental impact of the continuum to be demonstrated. In this paper, the biases between the above different formulae are used as a measure of their uncertainty.

[18] Figures 4 and 5 show water vapor and temperature Jacobians, respectively, calculated by using the LBL model with different water vapor continuum formulations. The curves calculated with continuum absorption have much larger sensitivity values than the NOCONT curves. The vertically integrated sensitivity is shown in Tables 1 and 2 . These numbers represent the change of OLR when the temperatures or water vapor concentrations are perturbed at all model layers simultaneously. Different continuum formulations give very similar values (in general, the relative bias, defined as $\left|\frac{\mathrm{CKD} 2.4-\mathrm{CKD} 2.1 \text { (or RSB) }}{\mathrm{CKD} 2.4}\right|$, is no larger than 5\%), although they differ from the NOCONT case substantially. The contrast between curves with continuum and the NOCONT curve is largest in lower and middle troposphere, especially in the TRP and MLS cases where water vapor is more abundant. Comparing Figure 6 (the spectral Jacobian of water vapor in tropical case calculated without continuum absorption) and Figure $2 \mathrm{a}$, it is apparent that the sensitivity is reduced most in the window region (the maximum decreases from more than $7.5 \mathrm{e}-4 \mathrm{~W} \mathrm{~m}^{-2} / \mathrm{cm}^{-1}$ to $\left.2.5 \mathrm{e}-4 \mathrm{~W} \mathrm{~m}^{-2} / \mathrm{cm}^{-1}\right)$. Thus, without continuum, OLR becomes very insensitive to the lower troposphere. The sensitivity is also reduced in the rotation band (the maximum decreases by $1 \mathrm{e}-4 \mathrm{~W} \mathrm{~m}^{-2}$ / $\mathrm{cm}^{-1}$ ), which makes the OLR less sensitive to middle troposphere. The change in $\nu 2$ band is less noticeable. The same decrease in these spectral regions also occurs for the spectral sensitivity to temperature (not shown here).

[19] The discrepancy between CKD2.1 and CKD2.4 exists only in middle and upper troposphere, which reflects the fact that they differ only in the parameters concerning the rotation band. Larger differences exist between RSB and the two CKDs. In terms of frequency-integrated Jacobians of water vapor (Figure 4), RSB gives larger values in lower and middle troposphere but smaller values in upper troposphere. In terms of frequency-integrated Jacobians of temperature (Figure 5), RSB over weights in the middle troposphere. The largest relative discrepancy at any model layer, though, is less than $10 \%$ for any case.

[20] Accurate LBL calculations show that ignoring continuum absorption may induce a few percent difference in simulated TOA radiative fluxes but larger difference in cooling rate [Clough et al., 1992]. Here, it is shown that 
(a) TRP

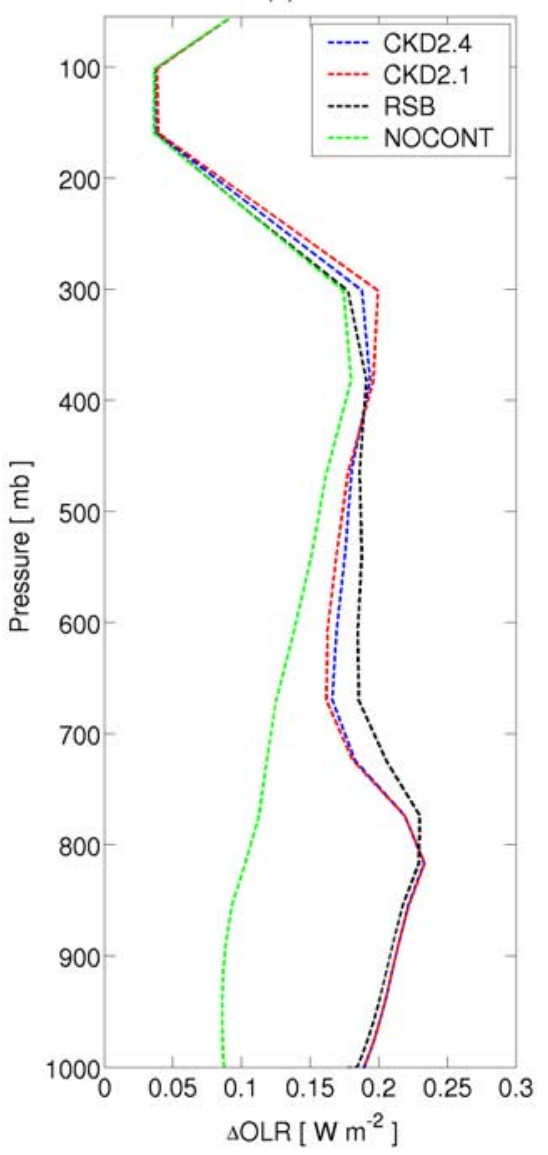

(b) MLS

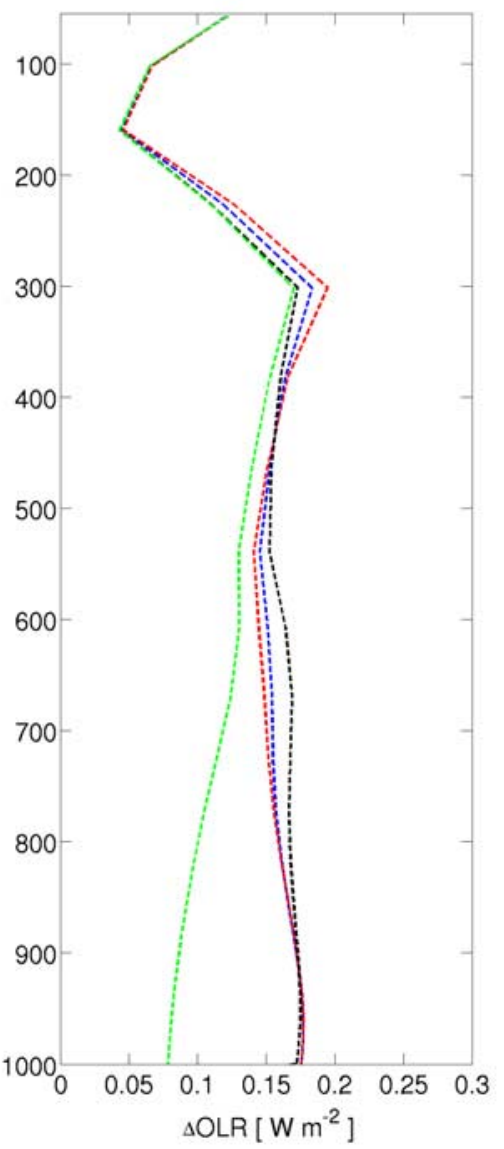

(c) MLW

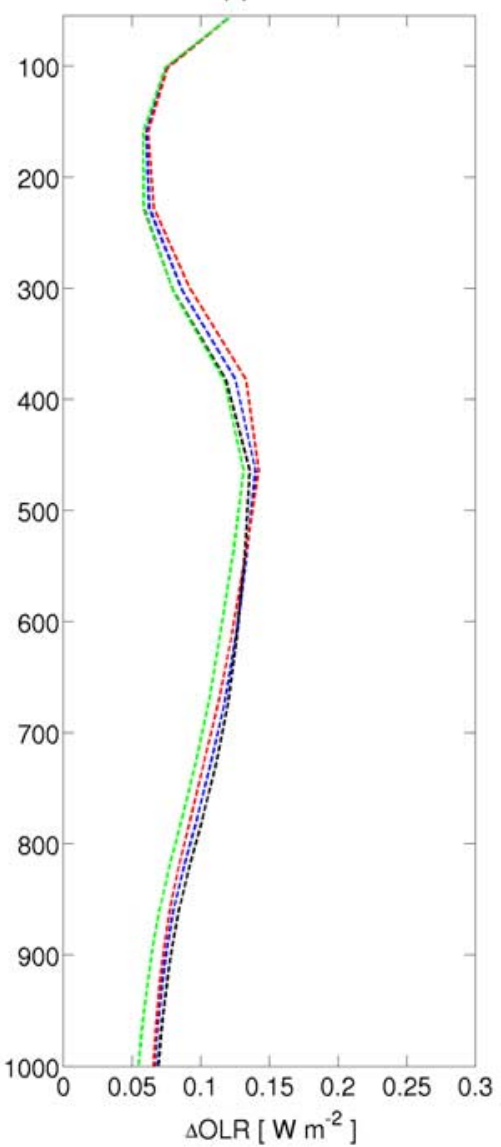

Figure 5. $(\mathrm{a}-\mathrm{c})$ Same as Figure 4 except for the Jacobians of temperature.

continuum may contribute up to $30-40 \%$ radiative sensitivity (i.e., Jacobian) of OLR (tropical case in Tables 1 and $2)$. Thus, as an extension of the earlier studies concerning radiative fluxes and cooling rates, the results here show that the water vapor continuum is indispensable and even more important for properly calculating the radiative sensitivities. However, the different empirical representations of the continuum induce relatively small differences indicating the current model simulations of water vapor feedback are not sensitive to the details of their continuum representation.

\subsection{Longwave Band Approximation}

[21] Another possible cause of uncertainty in the simulated OLR sensitivities is the longwave band approximation commonly used in climate models. In this paper, we examine the SEA method that is applied in the GFDL

Table 1. OLR Sensitivity to $10 \%$ Perturbation in Water Vapor Mixing Ratio in the Total Atmospheric Column ${ }^{a}$

\begin{tabular}{lccccc}
\hline & \multicolumn{5}{c}{ LBL } \\
\cline { 2 - 5 } & CKD2.4 & CKD2.1 & RSB & NOCONT & SEA, CKD2.4 \\
\hline TRP & 2.56 & 2.56 & 2.69 & 1.45 & 2.57 \\
MLS & 1.92 & 1.91 & 2.01 & 1.27 & 1.94 \\
MLW & 0.95 & 0.95 & 0.97 & 0.80 & 0.95 \\
\hline
\end{tabular}

${ }^{\mathrm{a}}$ Absolute value of the Jacobians is listed. Unit is $\mathrm{W} \mathrm{m}{ }^{-2}$. climate model's radiative transfer algorithm. The SEA method is a broad band approximation, with the longwave spectrum $\left(0-2200 \mathrm{~cm}^{-1}\right)$ divided into 8 frequency ranges [Schwarzkopf and Ramaswamy, 1999]. Results from the SEA method, as well as other longwave approximations, have been intensively examined over the ICRCCM project with respect to radiative fluxes and cooling rates. In this study, we extend this examination to radiative sensitivities, particularly the clear-sky OLR sensitivities to water vapor and temperature. Being derivatives, the radiative sensitivities (Jacobians) can be expected to be more sensitive functions of temperature and moisture.

[22] In Figure 7, the SEA approximation is compared to the benchmark LBL calculation. Both computations use the CKD2.4 continuum formulation. For the water vapor Jacobians, the LBL results overlap very well with the SEA

Table 2. OLR Sensitivity to 1 Kelvin Perturbation in Temperature in the Total Atmospheric Column ${ }^{\text {a }}$

\begin{tabular}{lccccc}
\hline & \multicolumn{5}{c}{ LBL } \\
\cline { 2 - 5 } & CKD2.4 & CKD2.1 & RSB & NOCONT & SEA, CKD2.4 \\
\hline TRP & 3.40 & 3.41 & 3.43 & 2.50 & 3.50 \\
MLS & 3.05 & 3.05 & 3.07 & 2.43 & 3.13 \\
MLW & 2.12 & 2.12 & 2.10 & 1.96 & 2.18 \\
\hline
\end{tabular}

${ }^{\mathrm{a}}$ Unit is $\mathrm{W} \mathrm{m}^{-2}$. 


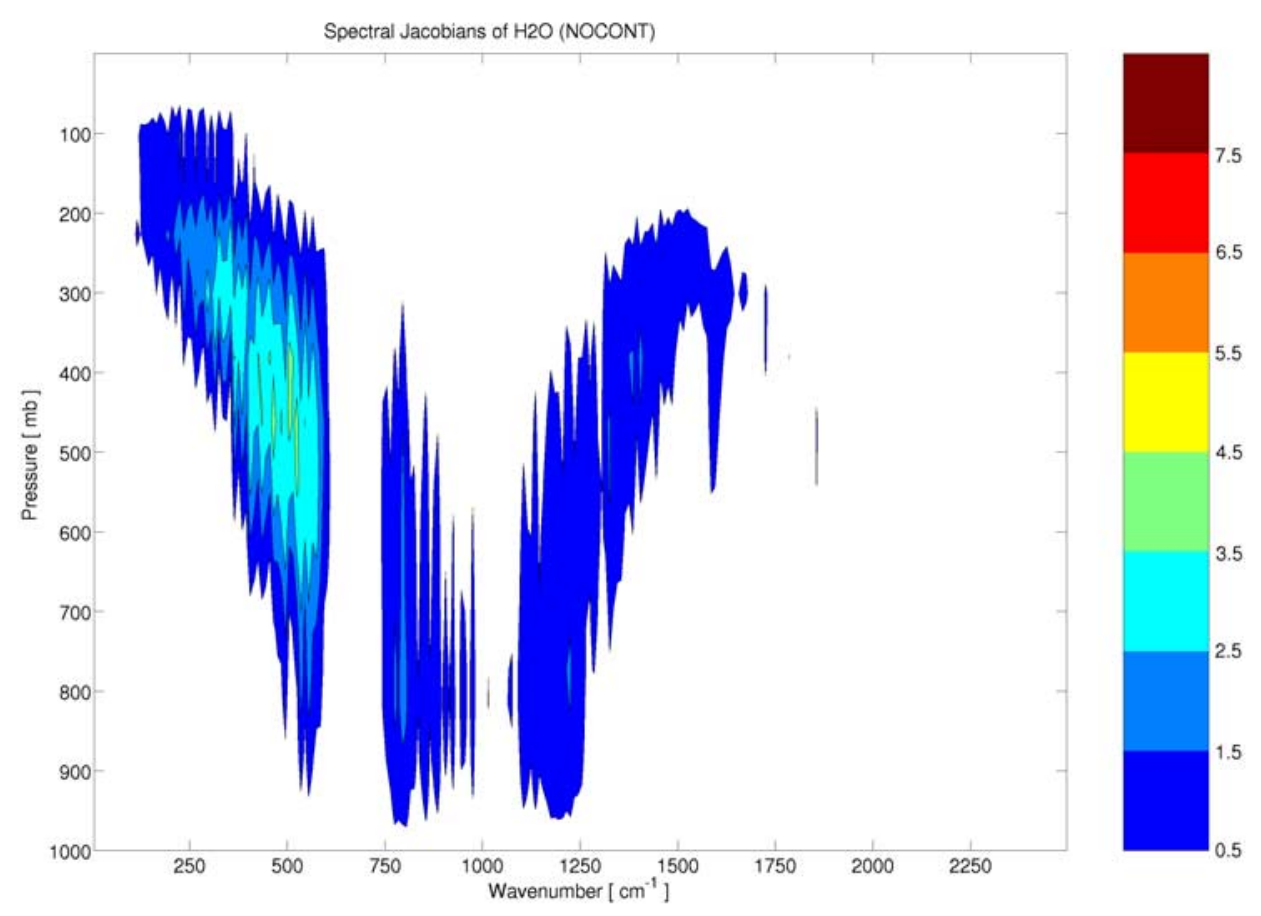

Figure 6. Spectral Jacobians for water vapor calculated without including continuum absorption, and plotted in the same manner as in Figure 2.

results for all the three atmospheres, indicating a very good agreement. Although the discrepancy of the temperature Jacobians seems to be larger, the relative error, taking the LBL as the reference, is well below $10 \%$ at any layer, except around tropopause in the tropical case.

[23] On the basis of the above results, we infer that, compared to the LBL results, the SEA approximation does not induce significant error in the clear-sky OLR sensitivities. This gains us confidence that climate models are capable of properly simulating clear-sky OLR as affected by either water vapor or temperature changes.

\section{3. "Cess-Type" GCM Experiment}

[24] The above results concerning the uncertainties due to water vapor continuum and longwave band approximation are obtained from radiative transfer calculations with static atmospheric profiles. It is of interest to know whether the conclusions could be affected if atmospheric conditions are allowed to change and interact with the radiation field, namely, in the context of climate feedbacks. To investigate this, a "Cess-type" GCM experiment [Cess et al., 1990] is conducted to infer the climate response to a SST perturbation. A description of these types of experiments is given by Soden et al. [2004]. We focus, again, on the clear-sky OLR. It is of our particular interest to examine whether the clearsky OLR response and the accompanying inverse climate sensitivity parameter (defined as the global mean clear-sky OLR difference divided by the global mean temperature difference, $\left[\mathrm{W} \mathrm{m}{ }^{-2} / \mathrm{K}\right]$ ) are markedly affected by differences in the continuum formulations.

[25] Four sets of GFDL GCM [GAMDT, 2004] integrations are performed over the period from 1982 to 1986. Different water vapor continuum schemes, namely, CKD2.4,
CKD2.1, RSB and NOCONT, are employed respectively in the four sets. In each set, there are two runs with prescribed sea surface temperature (SST), perturbed by $+2 \mathrm{~K}$ and $-2 \mathrm{~K}$ respectively. The same well-mixed greenhouse gas $\left(\mathrm{CO}_{2}\right.$, $\mathrm{CH}_{4}, \mathrm{~N}_{2} \mathrm{O}, \mathrm{CFC}-11$, CFC-12, CFC-113 and HCFC-22) concentrations, chosen arbitrarily as those corresponding to the year 1992, are used in all the integrations. The actual values of the well mixed greenhouse gases are immaterial in the context of this section.

[26] Figure 8 illustrates the geographical distribution of the 5-year mean clear-sky OLR difference between the two runs in each of the four groups, and lists the inverse climate sensitivity parameter. Very similar distribution of OLR differences are seen for the CKD2.4, CKD2.1 and RSB formulations. However, these three differ substantially from the NOCONT formulation. The OLR changes in the runs with continuum are much lower (up to $6 \mathrm{~W} \mathrm{~m}^{-2}$ ) than that in the NOCONT run. This is because the water vapor continuum absorption traps much of the increased outgoing longwave radiation resulting from the increased surface temperature. Without the continuum absorption, as illustrated by the previous comparisons of spectral Jacobians, the window region becomes highly transparent and the water vapor feedback is greatly reduced. In CKD2.4, CKD2.1 and RSB runs, the inverse climate sensitivity parameters are $2.06,2.05$, and $2.03 \mathrm{~W} \mathrm{~m}^{-2} \mathrm{~K}^{-1}$ respectively. They agree with each other to within $2 \%$, but differ by more than $20 \%$ from the NOCONT run $\left(2.56 \mathrm{~W} \mathrm{~m}^{-2} \mathrm{~K}^{-1}\right)$. This confirms that the inclusion of water vapor continuum absorption is indispensable for climate models to properly simulate climate sensitivity. However, different formulations of the continuum (including the neglect of the foreign broadening mechanism, as in the RSB scheme) induce only negligible 
(a) Water vapor Jacobians

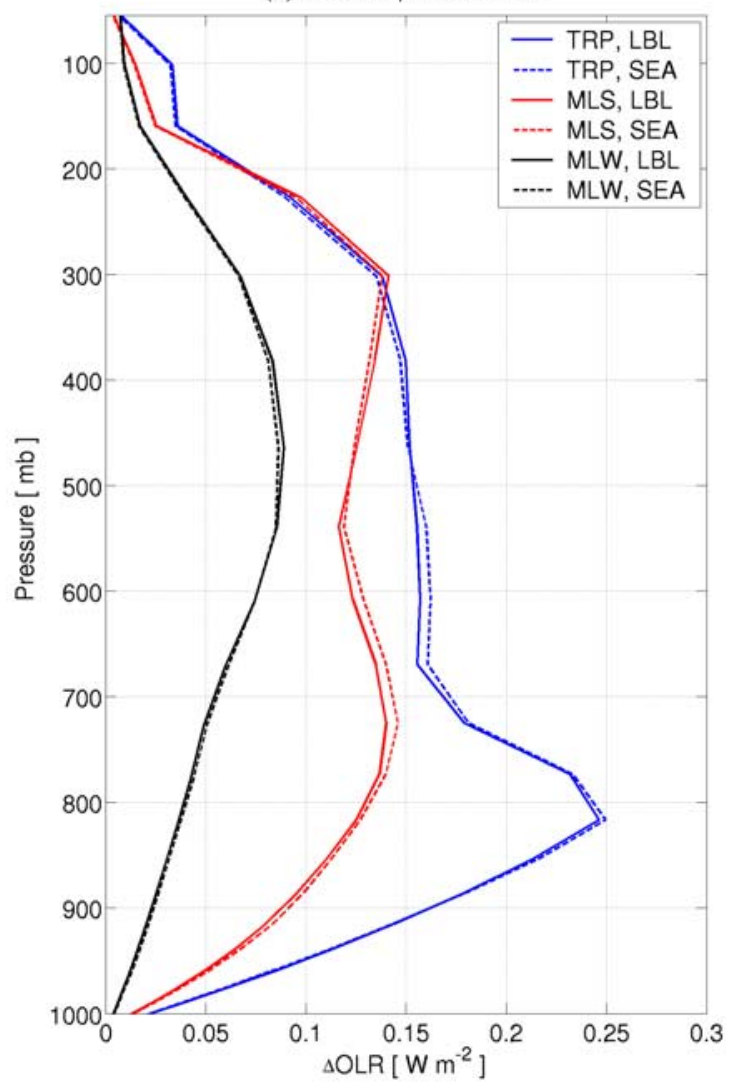

(b) Temperature Jacobians

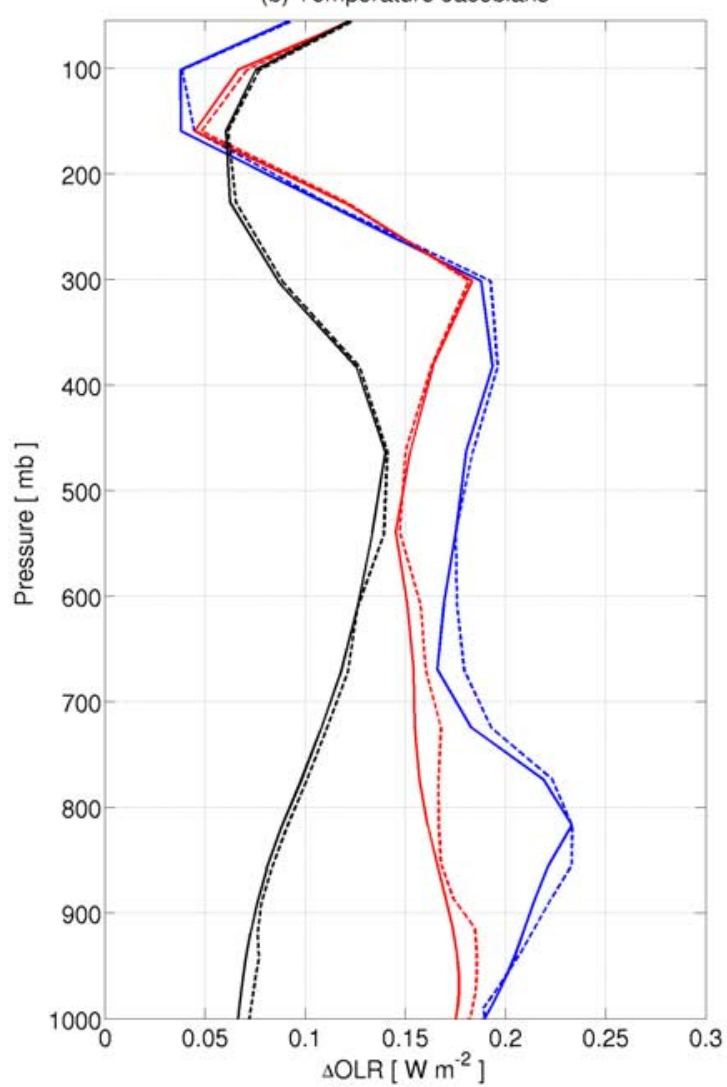

Figure 7. Comparison of the Jacobians calculated with the LBL model and SEA method for (a) water vapor and (b) temperature. The computation is performed for three atmospheres: TRP, MLS, and MLW.

difference in terms of clear-sky OLR response to SST change.

\section{Application of the Jacobians}

[27] Given the preceding analyses, if the residual term in equation (1) is small enough, the change of OLR can be approximately reproduced by linearly adding the products of the radiative Jacobians and the increments in temperature and water vapor. This gives us a first order estimate of the OLR change. Here we test the application of this idea with a GCM experiment. A 4-member ensemble of AM2 integrations from 1980 to 1999 are performed with observed SSTs (This is akin to an AMIP-type experiment [Gates et al., 1999] as conducted by GAMDT [2004]). The well-mixed greenhouse gas and ozone concentrations are fixed at the values of the beginning year (1980). Thus the only timevarying forcing is due to SST variations as prescribed from observations. The tropical (defined here as 30S-N) annual mean anomaly of SST, temperature profile and water vapor mixing ratio profile are obtained from the GCM integration. By multiplying the temperature and water vapor Jacobians for the tropical atmosphere and then adding the differentials, a time series of the clear-sky OLR anomaly is obtained. It is then compared to that explicitly calculated in the GCM integration, as illustrated in Figure 9. Actually, two different OLR anomaly series are derived from the temperature and water vapor Jacobians. OLR1 is reconstructed by using both temperature and water vapor Jacobians as in Figure 1; OLR2 is reconstructed by assuming conserved $\mathrm{RH}$ and using the Jacobians shown in Figure 3. The three results are generally in good agreement with each other, especially between the GCM simulation and OLR1. An examination of $\mathrm{RH}$ profile evolution of this period shows that the annual mean $\mathrm{RH}$ values indeed change only within very small extent $(<1 \%)$, except in some years which correspond to where relatively larger discrepancies occur. The good agreement between the reconstructed time series and the GCM simulation validates the use of the Taylor expansion as in equation (1), confirming that the temperature and water vapor contributions to clear sky OLR change are linearly additive.

[28] Reconstructing the OLR anomalies in this way, enables the isolation of the contributions to OLR change from different controlling factors. Figure 10 gives an example for 1989 (a strong La Nina year) and 1998 (a strong El Nino year). (The results are consistent for other years, except when the OLR anomaly signal is extremely small.) Figure 10a illustrates the attribution of the OLR anomaly to changes in the surface temperature, atmospheric temperatures and atmospheric water vapor concentrations. These correspond to the first three terms in equation (1), respectively. The contributions by atmospheric temperature and water vapor always drive the OLR anomaly in opposite 
a) $\mathrm{CKD} 2.4, \mathrm{dR} / \mathrm{dT}=2.06 \mathrm{~W} \mathrm{~m}^{-2} \mathrm{~K}^{-1}$

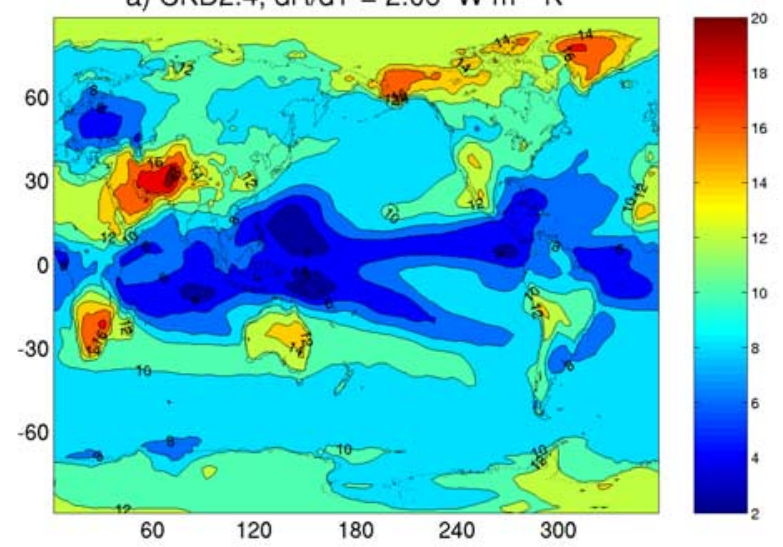

c) RSB, dR/dT $=2.03 \mathrm{~W} \mathrm{~m}^{-2} \mathrm{~K}^{-1}$

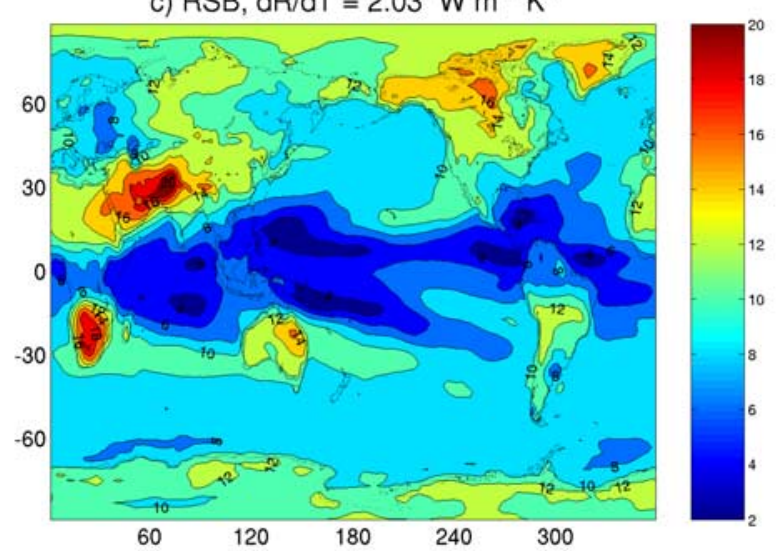

b) $C K D 2.1, \mathrm{dR} / \mathrm{dT}=2.05 \mathrm{~W} \mathrm{~m}^{-2} \mathrm{~K}^{-1}$

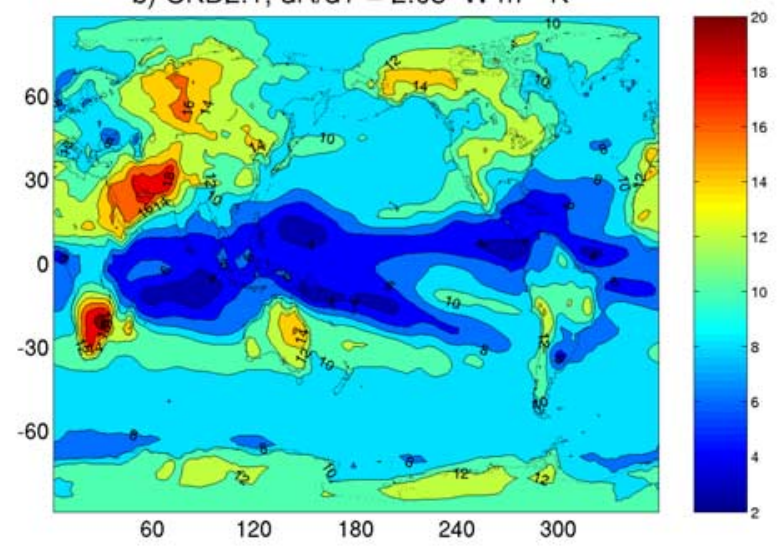

d) NOCONT, dR/dT $=2.56 \mathrm{~W} \mathrm{~m}^{-2} \mathrm{~K}^{-1}$

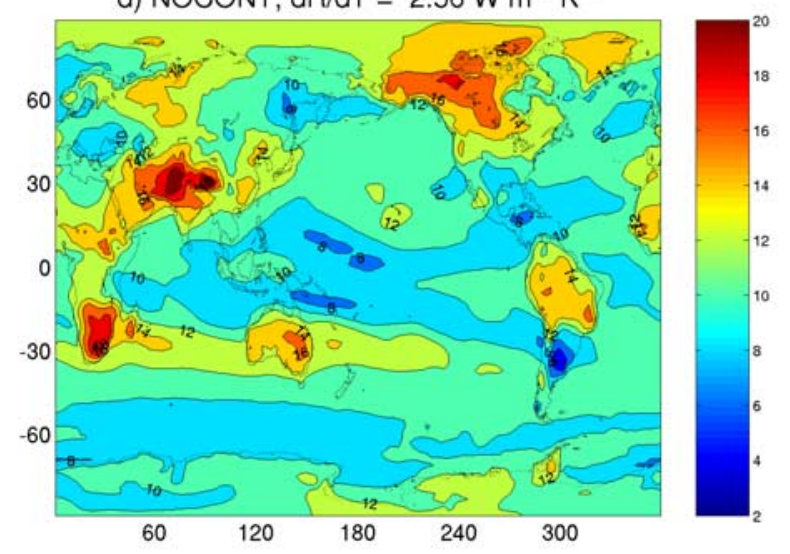

Figure 8. Climatological distribution of clear-sky OLR difference between the $+2 \mathrm{~K}$ and $-2 \mathrm{~K}$ prescribed SST runs for different continuum formulations. The inverse climate sensitivity parameter (see text) is also listed. (a) CKD2.4, dR/dT $=2.06 \mathrm{~W} \mathrm{~m}^{-2} \mathrm{~K}^{-1}$; (b) CKD2.1, dR/dT $=2.05 \mathrm{~W} \mathrm{~m}^{-2} \mathrm{~K}^{-1}$; (c) $\mathrm{RSB}, \mathrm{dR} / \mathrm{dT}=2.03 \mathrm{~W} \mathrm{~m} \mathrm{~K}^{-2} \mathrm{~K}^{-1}$; and (d) NOCONT, $\mathrm{dR} / \mathrm{dT}=2.56 \mathrm{~W} \mathrm{~m}^{-2} \mathrm{~K}^{-1}$.

directions. However, the temperature contribution dominates, so that the OLR anomaly has the same sign as the temperature contribution. This, as in the preceding constant RH case study, also suggests that, under clear sky conditions, the positive feedback of water vapor is smaller than the damping effect of temperature.

[29] The contribution by water vapor or temperature can be further attributed to different vertical portions of the atmosphere. In Figures $10 \mathrm{~b}$ and 10c, water vapor and temperature contributions are divided into four parts, respectively from stratosphere $(\mathrm{P}<100$ mbar), upper troposphere (100 mbar $<\mathrm{P}<400$ mbar), middle troposphere (400 mbar $<\mathrm{P}<700$ mbar) and lower troposphere $(\mathrm{P}>$ 700 mbar). The stratosphere is relatively less important for both water vapor and temperature contributions. For the water vapor contribution, the contribution from the upper troposphere is usually the largest because of stronger temperature variation there than the lower parts of the troposphere. This result, with upper troposphere being weighted more than the lower parts in terms of climate sensitivity to water vapor, agrees with other studies, such as Schneider et al. [1999].

[30] Concerning the temperature contribution, the upper, middle and lower troposphere contribute nearly equally. It is noted that, in upper troposphere, water vapor and temperature contributions are rather close in magnitude. If both contributions are added, there is a subtle cancellation. So it becomes difficult to determine the sign of the OLR change driven by upper tropospheric changes. This highlights the importance of studying upper tropospheric humidity and its variations.

[31] Here, the OLR reconstruction and feedback analysis have only been applied in the tropics. Because the seasonal variation of the atmosphere there is relatively small and the profiles are close to the standard tropical profile, the use of only one set of the radiative Jacobians (tropical) is justifiable. At higher latitudes, considering the interannual variation of the profiles, there is a need to apply different Jacobians for the different seasons. More rigorously we may compute the Jacobians seasonally and latitudewise, or even grid pointwise, as illustrated by Held and Soden [2000] 


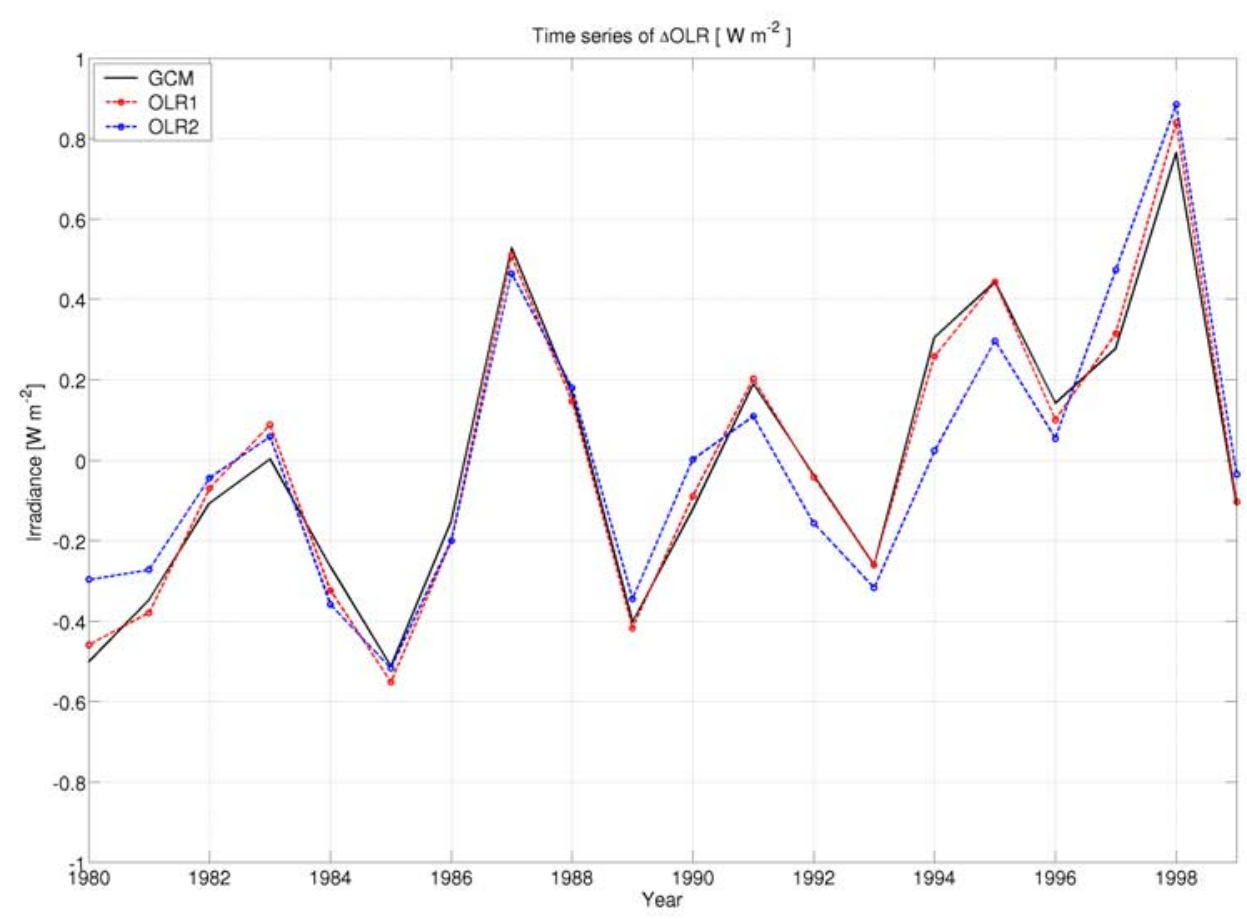

Figure 9. Temporal evolution of the tropical annual mean clear-sky OLR anomaly. "GCM" is calculated online using the GFDL GCM. "OLR1" is reconstructed by using both the water vapor and temperature Jacobians. "OLR2" is reconstructed by using the Jacobians under constant relative humidity condition.

(The adjoint method is a computationally efficient way to achieve this goal, e.g., Li and Navon [1998]). These Jacobians once computed will enable the feedback analysis to be done without running the offline radiation code and thereby make an efficient use of computational resources.

\section{Conclusion and Discussion}

[32] The radiative energy budget at TOA is crucial for climate studies. An important part of the budget, OLR, is governed by the surface and atmospheric temperatures, and various absorbers of longwave radiation. Under clear-sky condition, temperature and water vapor are two key variables controlling OLR. Here, we study the sensitivity of OLR to these two factors by investigating the radiative Jacobians, i.e., the partial derivatives of OLR with respect to temperature and water vapor. The Jacobians are calculated using both the GFDL LBL model and the GFDL GCM radiation code for three standard atmospheres (TRP, MLS and MLW). Different water vapor continuum absorption formulations are considered. The comparison of the simulated Jacobians shows that neither the longwave band approximation method (SEA), nor the different formulations of water vapor continuum absorption, cause significant difference in terms of clear-sky OLR sensitivity. Moreover, in a climate sensitivity experiment forced by prescribed global SST changes, it is demonstrated that water vapor continuum absorption is indispensable for correct simulation of the climate sensitivity, although the uncertainty introduced by different continuum formulations is small. It should be noted, though, that the agreements, in terms of Jacobians of clear-sky OLR, between different continuum representations and between longwave band approximation and LBL model do not guarantee the agreement in terms of other quantities. In fact, studies by Clough et al. [1992] and Schwarzkopf and Ramaswamy [1999] showed noticeable difference in calculated heating rates when applying different continuum representation schemes.

[33] Because the TOA radiation budget's response to atmospheric changes is critically important for climate change simulations, it is suggested that, in radiation code intercomparisons, there be a comparison of both the simulated irradiance fluxes as well as the accompanying radiative sensitivity, preferably in a spectral decomposition sense. This provides a strict test for radiation codes, particularly in quantifying climate feedbacks.

[34] By using the Jacobians of temperature and water vapor, the tropical clear-sky OLR change has been reconstructed from the surface and atmospheric conditions, instead of doing a full radiative transfer calculation. The reconstructed OLR time series is in very good agreement with that calculated online by the climate model. This encourages us to diagnose the water vapor and temperature feedback with the aid of these Jacobians. Thus, without running a climate model or merely running a radiative transfer code offline as done in many earlier studies, it is possible to obtain a measure of the altitude-dependent water vapor and temperature feedback. The result shows that the contribution to OLR change by temperature clearly exceeds that by water vapor in middle and lower troposphere. This is also demonstrated by an earlier example here that assumes constant relative humidity. The contribution to OLR change 
(a) Contributions to $\triangle \mathrm{OLR}$

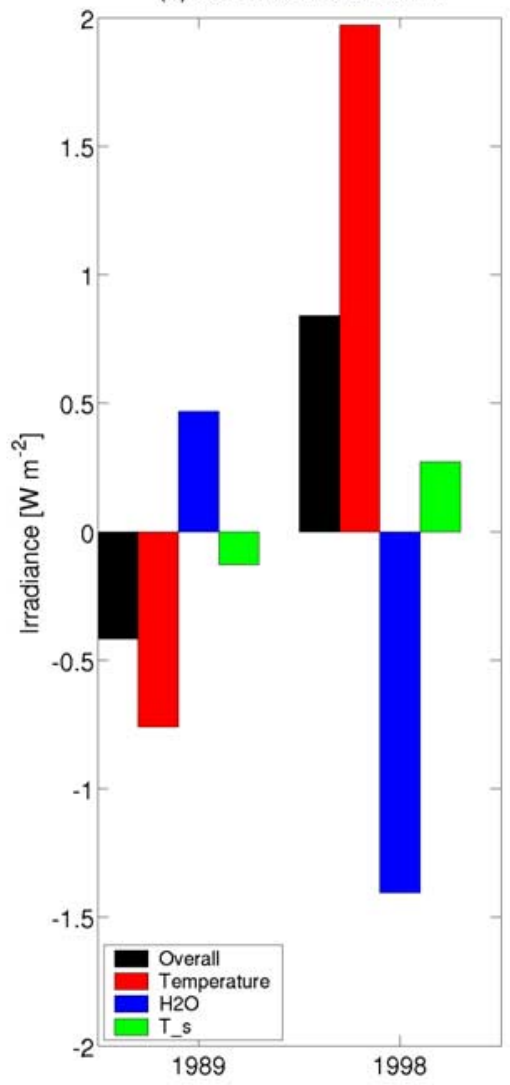

(b) Temperature contribution

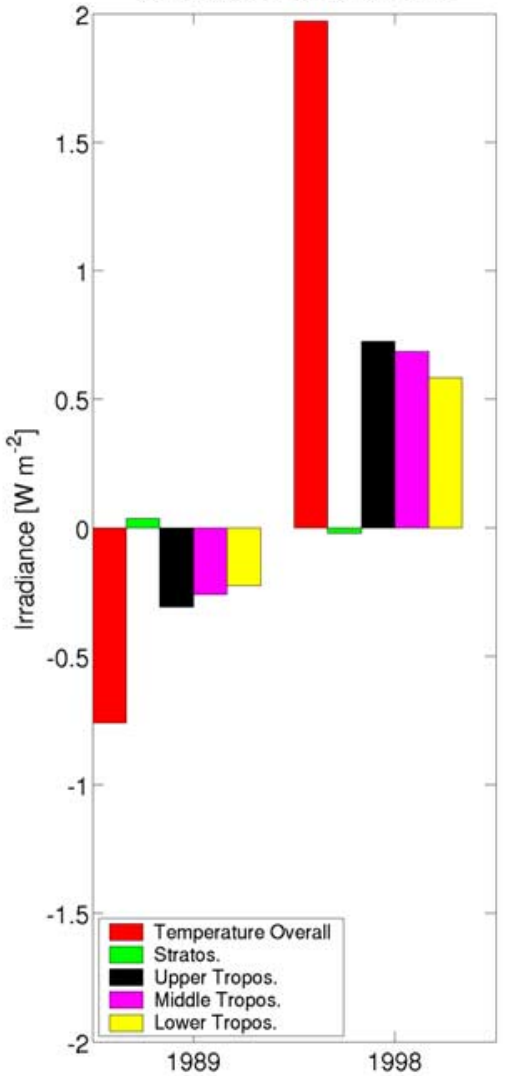

(c) Water vapor contribution

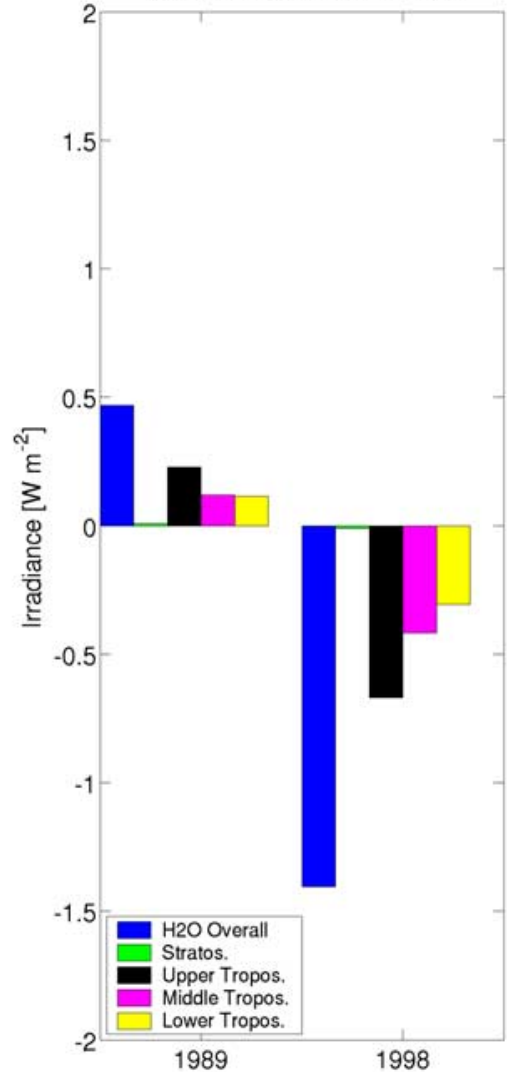

Figure 10. Temperature and water vapor contributions to the OLR anomalies. (a) Overall OLR change, i.e., the OLR anomalies as illustrated by "OLR1" in Figure 9, divided into the contributions by atmospheric temperature, atmospheric water vapor and surface temperature. (b) Overall contribution by temperature further attributed to different vertical parts of the atmosphere, i.e., stratosphere, upper troposphere, middle troposphere, and lower troposphere. (c) Vertical attribution made in terms of the contribution by water vapor.

from upper troposphere, where feedbacks of opposite nature by water vapor and temperature are in subtle cancellation with each other, becomes highly ambiguous. This reiterates the importance of studying upper tropospheric humidity's role in climate variation and change.

[35] The Jacobians have been calculated only for clearsky conditions. If clouds are introduced into the calculation, it is equivalent to adding a strong longwave absorber at specific layers in the vertical profile. Then, the OLR sensitivity to the lower troposphere will be reduced and the upper troposphere will have more weights in terms of the water vapor and temperature feedback. In the total sky situation, OLR is also sensitive to many properties of clouds, such as its height, coverage and liquid water amount. Dealing with cloud overlaps is a challenging issue [Sinha and Shine, 1995], and the assumption of linearity of OLR change as in equation (1) may break down because the variation of cloud amount and emissivity cannot be treated as small radiative perturbation. Thus the application based on Taylor expansion may be inappropriate for all-sky. However, the conceptual formalism of this work can be extended for clear-sky OLR sensitivities to other greenhouse gases besides water vapor.
[36] Acknowledgments. We thank Xianglei Huang, Dan Schwarzkopf, and three anonymous reviewers who helped improve the quality of this paper.

\section{References}

Allan, R. P., et al. (1999), The dependence of clear-sky outgoing longwave radiation on surface temperature and relative humidity, $Q . J$. R. Meteorol. Soc., 125, 2103-2126.

Allan, R. P., V. Ramaswamy, and A. Slingo (2002), Diagnostic analysis of atmospheric moisture and clear-sky radiative feedback in the Hadley Centre and Geophysical Fluid Dynamics Laboratory (GFDL) climate models, J. Geophys. Res., 107(D17), 4329, doi:10.1029/2001JD001131.

Bony, S., and J. P. Duvel (1994), Influence of the vertical structure of the atmosphere on the seasonal-variation of precipitable water and greenhouse-effect, J. Geophys. Res., 99, 12,963-12,980.

Cess, R. D., et al. (1990), Intercomparison and interpretation of climate feedback processes in 19 atmospheric GCMs, J. Geophys. Res., 95, $16,601-16,615$.

Cess, R. D., et al. (1996), Cloud feedback in general circulation models: An update, J. Geophys. Res., 101, 12,791-12,794.

Clough, S. A., F. X. Kneizys, and R. W. Davies (1989), Line shape and the water vapor continuum, Atmos. Res., 23, 229-241.

Clough, S. A., M. J. Iacono, and J.-L. Moncet (1992), Line-by-line calculations of atmospheric fluxes and cooling rates: Application to water vapor, J. Geophys. Res., 97, 15,761-15,785.

Ellingson, R. G., and Y. Fouquart (1991), The intercomparison of radiation codes used in climate models: An overview, J. Geophys. Res., 96, 89258928 .

Fasullo, J., and D. Sun (2000), Radiative sensitivity to water vapor under all-sky conditions, J. Clim., 14, 2798-2807. 
Fels, S. B., and M. D. Schwarzkopf (1975), The simplified exchange approximation: A new method for radiative transfer calculations, J. Atmos. Sci., 32, 1475-1488.

Fels, S. B., and M. D. Schwarzkopf (1981), An efficient, accurate algorithm for calculating $\mathrm{CO}_{2} 15 \mu \mathrm{m}$ band cooling rates, J. Geophys. Res., 86, $1205-1232$.

Gates, W. L., et al. (1999), An overview of the results of the Atmospheric Model Intercomparison Project (AMIP I), Bull. Am. Meteorol. Soc., 80, $29-55$.

Gillett, N. P., M. R. Allen, and S. F. B. Tett (2000), Modelled and observed variability in atmospheric vertical temperature structure, Clim. Dyn., 16, 49-61.

Global Atmospheric Model Development Team (2004), The new GFDL global atmospheric and land model (AM2-LM2): Evaluation with prescribed SST simulations, J. Clim., 17, 4641-4673.

Held, I. M., and B. Soden (2000), Water vapor feedback and global warming, Annu. Rev. Energy Environ., 25, 441-475.

Li, Z., and I. M. Navon (1998), Sensitivity of the Earth's radiation budget in the NCEP medium-range forecasting model, J. Geophys. Res., 103, $3801-3814$.

Liou, K. N. (1980), Infrared Radiation Transfer in the Atmosphere, An Introduction to Atmospheric Radiation, 392 pp., Elsevier, New York.

McClatchey, R. A. (1972), et al., Optical properties of the atmosphere, Environ. Res. Pap. 411, Air Force Cambridge Res. Lab., Mass.

Raval, A., A. H. Oort, and V. Ramaswamy (1994), Observed dependence of outgoing longwave radiation on sea surface temperature and moisture, J. Clim., 7, 807-821.

Roberts, R. E., J. E. A. Selby, and L. M. Biberman (1976), Infrared continuum absorption by atmospheric water vapor in the $8-12$ micron meter window, Appl. Opt., 15, 2085-2090.

Schneider, E. K., B. P. Kirtman, and R. S. Lindzen (1999), Tropospheric water vapor and climate sensitivity, J. Atmos. Sci., 56, 1649-1658.

Schwarzkopf, M. D., and S. B. Fels (1985), Improvements to the algorithm for computing $\mathrm{CO}_{2}$ transmissivities and cooling rates, J. Geophys. Res., $90,10,541-10,550$.
Schwarzkopf, M. D., and S. B. Fels (1991), The simplified exchange method revisited: An accurate, rapid method for computation of infrared cooling rates and fluxes, J. Geophys. Res., 96, 9075-9096.

Schwarzkopf, M. D., and V. Ramaswamy (1999), Radiative effects of $\mathrm{CH}_{4}$, $\mathrm{N}_{2} \mathrm{O}$, halocarbons and the foreign-broadened $\mathrm{H}_{2} \mathrm{O}$ continuum: A GCM experiment, J. Geophys. Res., 104, 9467-9488.

Shine, K. P., and A. Sinha (1991), Sensitivity of the earth's climate to height dependent changes in the water vapor mixing ratio, Nature, 354, $382-$ 384.

Sinha, A., and K. P. Shine (1995), Simulated sensitivity of the Earth's radiation budget to changes in cloud properties, Q. J. R. Meteorol. Soc., 121, 797-819.

Slingo, A., and M. J. Webb (1997), The spectral signature of global warming, Q. J. R. Meteorol. Soc., 123, 293-307.

Soden, B. J., A. J. Broccoli, and R. S. Hemler (2004), On the use of cloud forcing to estimate cloud feedback, J. Clim., 17, 3661-3665.

Spencer, R. W., and W. D. Braswell (1997), How dry is the tropical free troposphere? Implications for global warming theory, Bull. Am. Meteorol. Soc., 78, 1097-1106

Tobin, D. C., et al. (1999), Downwelling spectral radiance observations at the SHEBA ice station: Water vapor continuum measurements from 17 to 26 micron, J. Geophys. Res., 104, 2081-2092.

Zhang, M. H., R. D. Cess, J. J. Hack, and J. T. Kiehl (1994), Diagnostic study of climate feedback processes in atmospheric GCMs, J. Geophys. Res., 99, 5525-5537.

Y. Huang, Program in Atmospheric and Oceanic Sciences, Princeton University, Princeton, NJ 08544, USA. (yi.huang@noaa.gov)

V. Ramaswamy, NOAA Geophysical Fluid Dynamics Laboratory, Princeton, NJ 08542-0308, USA.

B. Soden, Rosenstiel School for Marine and Atmospheric Science, University of Miami, Miami, FL 33149, USA. 